\title{
The orbitofrontal cortex, food intake and obesity
}

\author{
Lauren T. Seabrook, BSc; Stephanie L. Borgland, PhD
}

\begin{abstract}
Obesity is a major health challenge facing many people throughout the world. Increased consumption of palatable, high-caloric foods is one of the major drivers of obesity. Both orexigenic and anorexic states have been thoroughly reviewed elsewhere; here, we focus on the cognitive control of feeding in the context of obesity, and how the orbitofrontal cortex (OFC) is implicated, based on data from preclinical and clinical research. The OFC is important in decision-making and has been heavily researched in neuropsychiatric illnesses such as addiction and obsessive-compulsive disorder. However, activity in the OFC has only recently been described in research into food intake, obesity and eating disorders. The OFC integrates sensory modalities such as taste, smell and vision, and it has dense reciprocal projections into thalamic, midbrain and striatal regions to fine-tune decision-making. Thus, the OFC may be anatomically and functionally situated to play a critical role in the etiology and maintenance of excess feeding behaviour. We propose that the OFC serves as an integrative hub for orchestrating motivated feeding behaviour and suggest how its neurobiology and functional output might be altered in the obese state.
\end{abstract}

\section{Introduction}

The obesity pandemic is one of the biggest global health challenges. Obesity is a chronic illness that affects people regardless of gender, age, socioeconomic status or geographical location. ${ }^{1-3}$ Obesity is defined as a body mass index (BMI) greater than $30 \mathrm{~kg} / \mathrm{m}^{2}$ and is associated with multiple comorbid diseases such as type 2 diabetes, stroke, cancer, depression and anxiety. ${ }^{1,4}$ Historically, obesity rates have been low; ${ }^{5}$ however, over the past 35 years, there has been a rapid increase in obesity rates and comorbid diseases. ${ }^{1-3,5,6} \mathrm{Al}$ though other environmental factors such as decreased energy expenditure and increased costs of healthy foods contribute to weight gain, multiple meta-analyses have concluded that overeating high-caloric food is the primary contributing factor to obesity. ${ }^{5-7}$ The modern obesogenic environment is largely related to the availability of easy-to-access, low-cost, highly palatable and energy-dense food, and this is a considerable driver of overeating. ${ }^{5,6}$ Prepackaged, convenient, ultra-processed foods play on our innate liking of sugars, salts and fats, ${ }^{8}$ and are often eaten even when energy requirements have been met. ${ }^{9}$ Understanding the neurobiological mechanisms of reward and influence of cognitive control that lead to excessive food intake may generate new knowledge about why we overeat despite satiety, and it may also point to potential therapeutic interventions.

Multiple brain regions and interacting systems regulate the orexigenic and anorexigenic states. ${ }^{10,11}$ The canonical understanding of food intake has been driven by a body of work describing how the adipocyte-released cytokine leptin regulates the neural control of energy balance through its modulation of the melanocortin system that originates in the arcuate nucleus of the hypothalamus. ${ }^{12}$ Leptin influences the alterations in excitability of these neurons that differentially drive food intake. Activation of neuropeptide Y/agoutirelated peptide (AgRP) neurons drives food intake by AgRPmediated inhibition of melanocortin 4 receptors. Activation of proopiomelanocortin/cocaine- and amphetamineregulated transcript neurons releases $\alpha$-melanocyte stimulating hormone, an agonist of melanocortin 4 receptors, to suppress food intake. ${ }^{13}$ The arcuate nucleus of the hypothalamus receives blood-borne and neuronal signals that assess energy status and modulate the neural activity of these cells to achieve energy balance.

Food is also consumed for reasons other than balancing energy needs, such as stress eating, social eating and eating for pleasure. ${ }^{14}$ Importantly, the hypothalamic, mesolimbic, subcortical and prefrontal regions all interact to drive food

Correspondence to: S. Borgland, Department of Physiology and Pharmacology, University of Calgary, 3330 Hospital Dr NW, Calgary, AB T2N 1N4; s.borgland@ucalgary.ca

Submitted Sep. 25, 2019; Revised Nov. 30, 2019; Accepted Dec. 9, 2019; Publislhed online Mar. 13, 2020

DOI: $10.1503 / j p n .190163$ 
consumption (see reviews by Berthoud and Morrison, ${ }^{15}$ Kenny, ${ }^{16}$ and Timper and Brüning ${ }^{17}$ ). The mesolimbic dopamine system encodes cues that predict food availability and motivate people to engage in food-seeking. ${ }^{18}$ Access to palatable food can increase synaptic strength onto ventral tegmental area (VTA) dopamine neurons, an effect that drives increased food approach behaviours. ${ }^{19}$ In an environment with ready access to highly palatable, energy-dense food, the cognitive control of eating behaviour plays a dominant role in regulating body weight. For example, tasks involving response inhibition (such as the stop signal or Stroop tasks), decision-making tasks (such as the Iowa Gambling Task) and the relative reinforcing value of food task have a highly consistent relationship with BMI and eating behaviour, such that poorer performance on these tasks predicts higher BMI. ${ }^{20}$ Thus, brain circuits in the frontal cortex involved in response inhibition, decision-making and reward valuation also play key roles in modulating food intake. The role of the medial prefrontal cortex (mPFC) in feeding has been evaluated in other reviews, ${ }^{21}$ but the potential role of the orbitofrontal cortex (OFC) in ingestive behaviour and sensitivity to obesity has not received as much attention. This review will focus on the OFC and its role in obesity and feeding behaviour.

\section{Anatomy and circuitry of the OFC in response to diet}

In rodents, the OFC is located dorsally to the rhinal sulcus and rostrally adjacent to the agranular insular areas. It can be subdivided into the ventral, ventrolateral, lateral, medial and dorsolateral anatomic distinctions based on relative location to the ventral midline (Fig. 1). ${ }^{22,23}$ In humans, there is some inconsistency in the anatomic borders of the OFC, but this structure can broadly be classified as Brodmann areas 10, 11 and $47.2^{22,23}$ Subregions of the OFC in human and nonhuman primates are similar, but the rodent OFC does not contain a granular layer. ${ }^{24,25}$ In fact, the rodent OFC consists of agranular cortical regions, meaning that these regions lack a granular layer of small pyramid-shaped neurons in the middle cortical layers. The cortical column consists of layers I to VI. In layer II of the medial OFC, the cells are spread more homogeneously compared with the unevenly distributed layer II cells of the prelimbic regions of the $\mathrm{mPFC}$, which has a densely packed layer I. ${ }^{26}$ Furthermore, the lateral OFC is easily differentiated between layers I and II/III in contrast to the ventrolateral OFC, which has an even distribution of pyramidal neurons. ${ }^{26}$

Similar to other cortical regions, the OFC comprises inhibitory interneurons that synapse onto glutamatergic pyramidal neurons in layer II/III. ${ }^{27}$ These interneurons can be further classified as parvalbumin-positive $(\mathrm{PV}+)$, somatostatin, vasoactive intestinal polypeptide or cholecystokinin. ${ }^{28,29}$ The basket-cell morphology of these interneurons form vast perisomatic synapses. ${ }^{30}$ Basket cells are highly branched interneurons that form axosomatic synapses, and are therefore well positioned to coordinate pyramidal firing. ${ }^{31}$ Indeed, PV+ basket neurons entrain principal neuron output via $\gamma$-frequency oscillations (25-100 Hz). ${ }^{32}$ The PV+ interneurons in the OFC have been implicated in cognitive flexibility, such that



Fig. 1: Afferent and efferent projections from subregions of the rodent orbitofrontal cortex. BLA = basolateral amygdala; $D L S=$ dorsolateral striatum; DMS = dorsomedial striatum; LH = lateral hypothalamus; IOFC = lateral orbitofrontal cortex; $\mathrm{MC}=$ motor cortex; $\mathrm{mOFC}=\mathrm{medial}$ orbitofrontal cortex; $\mathrm{NAC}=$ nucleus accumbens; $\mathrm{OC}=$ olfactory cortex; $\mathrm{SC}=$ somatosensory cortex; $\mathrm{STN}=$ subthalamic nuclei; $\mathrm{VC}=$ visual cortex; vOFC = ventral orbitofrontal cortex; VTA = ventral tegmental area. 
transgenic mice with reduced PV expression showed impaired performance on a reversal learning task in concordance with altered OFC neuronal activity. ${ }^{33}$ Several studies have suggested that diet can also influence PV+ expression. In adult rats, exposure to a cafeteria diet for 6 weeks (ad libitum or intermittent access) did not alter PV+ expression in the lateral OFC. ${ }^{34}$ However, in mPFC subregions, intermittent access to a high-fat, high-sucrose diet during the adolescent period in rats (a manipulation that caused a small weight increase over controls) decreased PV+ interneuron expression in the infralimbic cortex. ${ }^{35,36}$ Intermittent access to $10 \%$ sucrose alone also decreased PV+ expression in the prelimbic cortex of adolescent male rats, but not the infralimbic cortex. ${ }^{37}$ This finding suggests that there may be differences in the influence of diet on $\mathrm{PV}+$ expression in different cortical regions. Alternatively, the age of animals during diet exposure or duration of diet exposure may differentially influence PV+ expression.

Diet exposure can also influence $\gamma$-aminobutyric acid (GABA) release. An 8-week, high-fat diet decreased GABA in rat frontal cortex homogenates measured with highperformance liquid chromatography. ${ }^{38}$ In the lateral OFC, obesity - but not limited access to diet - decreased GABAergic release probability onto layer II/III pyramidal neurons as measured by a decrease in the frequency of GABAergic miniature release events, as well as a paired pulse facilitation in slice electrophysiology recordings. ${ }^{34}$ Because $\mathrm{PV}+$ expression was not different in the lateral OFC of these obese rats, other interneurons or GABAergic inputs to lateral OFC pyramidal neurons may underlie decreased GABAergic synaptic transmission onto lateral OFC pyramidal neurons. ${ }^{34}$ Another proposed mechanism is that perineuronal nets influence GABAergic synaptic transmission. Perineuronal nets are extracellular matrix structures surrounding PV+ interneurons that can regulate synaptic plasticity. ${ }^{39}$ The expression of perineuronal nets can be influenced by diet. Exposure to a high-fat diet, independent of weight gain, decreased the number of perineuronal nets in the ventral OFC. ${ }^{40}$ Similarly, adolescent exposure to a high-fat, high-sucrose diet decreased expression of perineuronal nets in the infralimbic cortex of the mPFC. ${ }^{36}$ Taken together, GABAergic signalling in the OFC and $\mathrm{MPFC}$ may be especially sensitive to obesogenic diets and may influence cognitive flexibility.

The OFC pyramidal neurons also exhibit changes with an obesogenic diet. Decreases in basilar spines of lateral OFC pyramidal neurons have been found with an increase in branching of the basilar dendrites. ${ }^{34}$ In contrast, no changes were observed in apical spines or dendritic branching. Notably, basal and apical spine density is decreased in the prelimbic and infralimbic cortices after 3 weeks of exposure to a high-fat diet. ${ }^{41}$ Taken together, diet-induced obesity alters OFC cellular structure and function, and these changes may differ from those occurring in the $\mathrm{MPFC}$.

\section{Neural correlates of OFC function}

The OFC encodes value in an identity-specific manner. This specificity ensures that a decrease in the value of food after a meal does not affect the value of other rewards, such as protection from predators or reproduction. For example, popula- tions of OFC neurons initially respond to either sucrose (sweet taste) or quinone (bitter taste). As animals learn taskassociated outcomes, these neurons fire in anticipation of the taste, and then to cues associated with the palatable or unpalatable food. ${ }^{42}$ Interestingly, these neurons continue to fire even after these contingencies are heavily learned ${ }^{43}$ and do not scale if the reward is delivered or withheld unexpectedly. ${ }^{44}$ Thus, OFC neurons appear to fire in response to representations of expected outcomes. ${ }^{42-45}$ This is consistent with OFC activation in response to anticipatory events of a preferred food. ${ }^{45,46}$ A recent study demonstrated that VTA-projecting OFC neurons had stable calcium activity recorded in individual neuronal clusters in response to food-predicting cues that lasted after extinction of the cue-reward association. ${ }^{47}$ When single OFC neurons that previously responded to presentation of caloric rewards were selectively stimulated, mice increased licking responses to sucrose. ${ }^{48}$ Interestingly, when single OFC neurons that respond to social reward were selectively stimulated, mice decreased licking for sucrose, ${ }^{48}$ suggesting that OFC neurons appear to maintain their activity-specific representations. In addition, OFC activation can scale with hunger ${ }^{49-51}$ and the pleasantness of food rewards. ${ }^{45}$ Increased in vivo neuronal activation in the OFC occurs when hungry compared to when sated in rats, monkeys and humans. ${ }^{49-52}$ Taken together, these findings show that OFC neuronal activity encodes representations of outcomes and their subjective value in an identity-specific manner. Thus, disruption of the OFC with obesity or other comorbid disorders may result in altered value representations of foods.

\section{Afferent and efferent projections of the OFC}

The OFC integrates afferent and efferent projections, including sensory, limbic and prelimbic regions, to guide decision-making associated with food intake. Afferent projections include sensory inputs from the gustatory, olfactory and visual corti$\operatorname{ces}^{22,23}$ and use these multisensory modalities to modify behavioural output. In rodents, the olfactory bulb is located anterior to the OFC, and via the shortest pathway, pyramidal neurons in the OFC are 3 synapses distant from olfactory sensory neurons. This is unique, because other sensory modalities (such as visual or gustatory) pass through multiple relay centres before reaching the prefrontal cortex. ${ }^{53}$ The OFC receives gustatory cues, and it has been demonstrated that OFC neurons encode information about sweetness intensity. ${ }^{54}$ The OFC also responds to sensory characteristics of stimuli, including the flavour, appearance and texture of the reward. ${ }^{55}$

The OFC receives input from other parts of the prefrontal cortex, including motor and premotor regions, and from limbic projections, including the basolateral amygdala and the VTA. ${ }^{55-57}$ These inputs can segregate based on OFC subregion (Fig. 1). The medial OFC receives inputs from the thalamus, and the lateral OFC receives strong inputs from the amygdala. ${ }^{27}$ The central zone of the lateral and ventral OFC receives dopaminergic input from the VTA to guide adaptive response to changing outcome value and prediction error learning. ${ }^{44}$ In vivo calcium imaging of OFC neurons projecting to the VTA demonstrated increased activity in relation to 
long-term cue-reward memory representations of rewardseeking behaviour. ${ }^{47}$ Furthermore, decreasing the activity of dorsal-striatum-projecting OFC neurons reduces compulsivelike behaviour in mice trained to press a lever for optogenetic stimulation of VTA dopaminergic neurons despite a shock punishment. ${ }^{58}$ These data suggest that reciprocal projections between the OFC and the VTA guide reward-seeking behaviour, and could be modulated by obesogenic diets to bias food intake.

The basolateral amygdala and OFC circuit share dense reciprocal projections that support outcome-guided behaviour. ${ }^{55,56}$ Outcome-guided behaviour is the scaling of action based on the value of the outcome. For example, if the value of the reward decreases, action to receive that reward will also decrease. One of the primary roles of the basolateral amygdala is to assign positive or negative valence to stimuli by forming associations between neutral cues with awarding or aversive properties, helping to guide consumption. ${ }^{59}$ Thus, the OFC integrates sensory and limbic inputs to help optimize actions.

The OFC can be further subdivided into anterior and caudal areas, such that the anterior posterior OFC projects to the dorsolateral prefrontal cortex, insula and thalamus, whereas the caudal OFC projects to the thalamus and amygdala. ${ }^{43,60}$ The lateral OFC also sends strong projections to the dorsal medial and dorsal lateral striatum, ${ }^{58,61,62}$ whereas the medial OFC projects to the nucleus accumbens ${ }^{23}$ and the basolateral amygdala. ${ }^{43}$ The OFC concurrently projects to the lateral prefrontal cortex, which connects widely to motor and premotor areas $^{63}$ that could be important in guiding outcome responses. ${ }^{64}$ Thus, the OFC is positioned to influence a variety of functions, including decision-making and action selection of food and rewards.

\section{OFC and reward-seeking behaviour in lean animals}

Instrumental (operant) learning guides specific actions (e.g., lever presses) based on the rewarding consequence of the outcome (e.g., delivery of food in a better-than-expected context). This type of learning is based on flexible contingencies in which actions increase when a desirable outcome is to be achieved and decrease for a less desirable outcome or to avoid harmful and aversive outcomes. ${ }^{65}$ Goal-directed behaviour is a contingent relationship of value and associated outcome. It applies flexible learning, such that interaction is based on the reinforcing or aversive outcome value and is flexible in the face of changing contingencies. For example, goal-directed rodents will adapt their behaviour to maximize a desirable outcome. In contrast, habitual behaviour is insensitive to decreased current values of the outcome. For example, manipulation of the outcome (making the reward less valuable or even aversive) has no immediate effects on behaviour, such that animals continue to respond regardless of adverse or poorly optimized consequences. ${ }^{43,65}$ Appropriate decision-making requires integration of previous memories and outcomes from similar circumstances. ${ }^{43}$ The shift from goal-directed behaviour to habitual behaviour has been implicated in many disease states, including maladaptive decision-making, obsessive-compulsive disorder, eating disorders and addiction. ${ }^{66-68}$

The OFC is essential to the decision-making process, because it estimates the likelihood of a specific outcome to guide future responses. This estimation can be tested using outcome devaluation tasks. Outcome devaluation is a type of instrumental conditioning that can test goal-directed and habitual behaviour. Typically, animals will decrease a cueevoked response or action if the outcome (usually food) is devalued by satiation with the same type of food or by pairing the food with sickness using lithium chloride in a conditioned taste-aversion paradigm. Alternatively, the action to retrieve the reward (lever press) can be devalued by changing the contingency of the lever, so that action at the lever is no longer required to obtain a reward, and the animal must withhold response. This reversal of contingency requires the learning of new rules to obtain the reward. The knowledge that the OFC is critical in encoding the current value of rewards is supported by lesion and inactivation studies. Inactivation or lesions of the OFC after learning the cue association and before testing has been implicated in the devaluation of food rewards by sickness, ${ }^{69,70}$ satiety ${ }^{61,71,72}$ and contingency degradation tasks. ${ }^{73}$ The OFC has also been implicated in reversal learning, ${ }^{43,59}$ whereby animals must ignore their previously learned strategy and learn new rules to obtain a reward.$^{74}$ Finally, temporary inactivation of the OFC after training and stimulus-selective satiety before testing prevented accurate reward devaluation in a Pavlovian task. ${ }^{61}$ Based on these studies, it has been proposed that the OFC is responsible for updating the values of rewards and using this learned representation of outcomes to guide behaviour. ${ }^{59}$ Outcome expectancy relies on predictive cues that guide behaviour and the memory of what those cues predicted. With predictive cues guiding real-time behavioural computations and memory, the correct application of previous experiences can be applied to new situations. ${ }^{49}$ Outcome expectancy can provide information for real-time learning so that future behaviour is adaptive. Because outcome expectancy is often studied in the context of food and reward, ${ }^{57}$ it likely plays a key role in the adaptive regulation of food intake.

The OFC supports goal-directed behaviour, likely through its projections to the dorsomedial and dorsolateral striatum. ${ }^{61}$ The dorsomedial striatum has also been implicated in goaldirected behaviour: lesions in this brain region result in habitual behaviour. ${ }^{75-78}$ In comparison, the dorsolateral striatum modulates habitual behaviour: lesions in this brain region result in a shift to goal-directed behaviour. ${ }^{76-78}$ Conditioned taste aversion and selective satiety outcome devaluation are dependent on multiple brain structures, including the amygdala, the gustatory cortex and the OFC. ${ }^{43,79}$ Lesions in the gustatory cortex and amygdala inhibited both the acquisition and retrieval of outcome devaluation. ${ }^{79}$ In comparison, lesions in the OFC impaired cue-induced devaluation of sucrose when paired with lithium chloride, but not initial learning. ${ }^{71}$ Importantly, OFC lesions do not impair Pavlovian or instrumental learning; rather, acquisition of conditioned taste aversion is dependent on the basolateral amygdala, and 
choice decisions require the OFC. ${ }^{42,43}$ Consistent with this finding, pharmacological inactivation of the OFC impaired choice tests in outcome devaluation, but not the retrieval of the memory. ${ }^{69}$ Inactivation of the OFC does not change the perceived palatability of food rewards. ${ }^{80}$ Taken together, these findings show that the involvement of the OFC in outcome devaluation may serve to integrate reward-predictive cues and the new value of the rewards. In the context of feeding, the OFC integrates sensory information from consumption experience and performs computations to establish appropriate food-approach behaviour.

\section{OFC and reward-seeking behaviour in obese animals}

It has been proposed that goal-directed and flexible behaviour is impaired with obesity. Rats exposed to an unlimited cafeteria diet for 6 weeks ${ }^{81}$ experienced disruption in a Pavlovian devaluation task (Table 1). Rats were trained to associate 2 cues with distinct foods. Then, one of the foods was devalued with sensory-specific satiety. Rats on a cafeteria diet responded equally to both food predictive cues. The authors concluded that consumption of a cafeteria diet impaired stimulus-outcome learning and cued food associations. ${ }^{81}$ However, because cafeteria-fed rats decreased consumption of both valued and devalued food after selective satiation, others have argued that satiety-induced devaluation was not effective in selectively reducing the value of the pre-fed food in the cafeteria-fed rats; the deficit observed may not have reflected impaired stimulus-outcome learning, and instead may have reflected the insensitivity of the cafeteria-fed rats to selective satiety-induced devaluation. ${ }^{100}$ An alternate outcome devaluation method for testing this hypothesis would be to use a procedure that avoids reliance on satiety, such as lithium-chloride-induced devaluation (conditioned taste aversion). ${ }^{100}$ In another study, rats with 5 weeks of restricted access to sweetened condensed milk displayed a similar disruption in outcome devaluation, whereby rats with restricted access responded similarly to the undervalued and devalued reward conditions. ${ }^{99}$ Notably, rats with continuous access to the palatable food consumed fewer calories than those with restricted (binge-like) access but did not have deficits in outcome devaluation, suggesting a relationship with caloric intake and impairment in devaluation. ${ }^{99}$ Furthermore, in contrast to the findings of Reichelt and colleagues, ${ }^{81}$ the diet had no effect on sensory-specific satiety when the foods were freely available, suggesting that the deficit in reward devaluation in binge-access rats was associated with impaired stimulus-outcome learning rather than differences in satiety. ${ }^{99}$ Rats exposed to a high-fat diet for 3 months also exhibited insensitivity to devaluation, but only if they were trained on a random interval schedule, whereby rewards were delivered in response to random numbers of lever presses that facilitated habitual response. ${ }^{101}$ These findings might suggest that energy-dense diets affect sensitivity to devaluation by facilitating a transition to inflexible response in situations employing behavioural strategies that are highly sensitive to outcome devaluation. In contrast to this idea, using an outcome devaluation task that discouraged habitual performance, whereby actions were performed without consideration of their consequences, rats given 6 weeks of exposure to a junk-food diet demonstrated impaired satietyinduced outcome devaluation. ${ }^{98}$ Thus, rats continued to demonstrate impairment in the selection between 2 distinct food-seeking actions when one of the food outcomes was devalued without the development of habit-like behaviour.

Another method to test whether diet exposure impairs cognitive control over eating or alterations in stimulus-outcome learning is to use a Pavlovian Instrumental Transfer task. ${ }^{100,102}$ This task tests whether food-predicting cues can invigorate response for a cue associated with a specific food in rats exposed to control or palatable food diets. ${ }^{100,102}$ To further test for impairments in stimulus-outcome learning with junkfood diets, Kosheleff and colleagues ${ }^{98}$ tested the hypothesis that junk-food diets would impair control over specific foodseeking actions even when food values were not the primary basis for decision-making. Rats failed to use cue-elicited food expectations to guide their selection of actions based on the specific foods they represented in the Pavlovian Instrumental Transfer task. In other words, rats on the junk-food diet were

Table 1: Comparison of human and rodent behavioural performance and alterations in OFC structure and function

\begin{tabular}{|c|c|}
\hline Diet/species & Anatomic/functional changes \\
\hline Obese/human OFC & $\begin{array}{l}\text { Decreased OFC grey matter }{ }^{82,83} \\
\text { Decreased total OFC volume }{ }^{84} \\
\text { Altered fluid distribution in a diffusion-weighted image } \\
\text { MRI }{ }^{85} \\
\text { Increased and persistent BOLD imaging in the OFC in } \\
\text { response to rewarding food cues } \\
86,87,88\end{array}$ \\
\hline Binge-eating disorder/human OFC & $\begin{array}{l}\text { Increased BOLD to palatable food cues in the } \text { mOFC }^{93-95} \\
\text { Increased OFC grey matter volume }\end{array}$ \\
\hline Obese/rodent OFC & $\begin{array}{l}\text { Decreased GABA release and decreased basilar spines of } \\
\text { IOFC pyramidal neurons, with increased in branching of } \\
\text { the basilar dendrites } \\
\text { Decreased perineural nets in vOFC }{ }^{40}\end{array}$ \\
\hline Restricted diet (binge-like)/rodent OFC & Decreased perineural nets in vOFC ${ }^{40}$ \\
\hline
\end{tabular}

Behavioural changes

Decreased satiety-induced reward devaluation ${ }^{89}$ Decreased response inhibition in the Stroop task ${ }^{90,91}$ Increased errors in modified food go/no-go task ${ }^{92}$

Eating restraint inversely associated with BOLD in OFC during the Stroop task ${ }^{97}$

Disrupted reward devaluation via selective satiety ${ }^{81}$ Disrupted cue-evoked reward expectations ${ }^{98}$

Disrupted reward devaluation via selective satiety ${ }^{99}$ Disrupted cue-evoked reward expectations ${ }^{98}$

$\mathrm{BOLD}=$ blood-oxygen-level-dependent; $\mathrm{GABA}=\gamma$-aminobutyric acid; IOFC $=$ lateral orbitofrontal cortex; $\mathrm{mOFC}=$ medial orbitofrontal cortex OFC $=$ orbitofrontal cortex vOFC $=$ ventral orbitofrontal cortex. 
impaired in selecting actions based on expected food value or the presence of food-paired cues, ${ }^{98}$ suggesting that energydense or palatable diets can disrupt cognitive control over feeding. Future work should test how the OFC is implicated in these diet-induced changes to flexible behaviour.

\section{OFC and obesity in humans}

Recent neuroimaging studies in humans are beginning to elucidate the complexity of the role of the OFC in the behavioural and neurobiological phenotypes that underlie obesity (Table 1). Structural and volumetric differences can be observed in the OFC related to body weight. People who are obese or morbidly obese have decreased OFC grey matter volume, ${ }^{82,83}$ decreased total OFC volume ${ }^{84}$ and altered fluid distribution in diffusion-weighted imaging. ${ }^{85}$ These structural differences have been implicated in altered executive function. ${ }^{83}$ Functional imaging demonstrates that the OFC, as well as other structures involved in reward processing, are activated in response to food cues (see van der Laan and colleagues ${ }^{103}$ ), and people who are obese show increased blood oxygen-level-dependent (BOLD) activity in brain regions that encode cognitive control and reward (see Lowe and colleagues ${ }^{21}$ ). Obese individuals showed increased activation of the OFC in response to visual food cues than leaner individuals. ${ }^{86}$ Interestingly, this activation persisted even when people were sated, suggesting that people who are obese appear to be more responsive to food cues when sated than lean individuals. Notably, increased BMI predicts OFC activation in response to food images. ${ }^{104,105}$ Consistent with this, obese women who fasted for 8 to 9 hours showed greater activation in the OFC, nucleus accumbens, anterior cingulate cortex and $\mathrm{mPFC}$ in response to images of highly palatable, high-calorie food compared with images of neutral or lowcalorie food. ${ }^{87}$ It has been proposed that obesity may be linked to increased neural responses in reward anticipation from food cues, but decreased responses during food consumption. ${ }^{88}$ Activation of the OFC in response to food cues in obese people regardless of satiety may underlie their vulnerability to overeating and diet failures.

Similar to rodents, the human OFC shows functional heterogeneity, such that the medial OFC encodes the value of food rewards, whereas the lateral OFC represents the nutritive encoding that is then integrated and processed in the medial OFC to compute overall value. ${ }^{106}$ Value encoding is the scaling of activation with the subjective value of the reward; this can be associated with food, monetary or other rewards. Nutritive encoding is activation based on perceived nutritive value, such that the subjective value of foods can be predicted by their perceived nutritive composition, including fat, carbohydrate, protein or vitamin content. ${ }^{106}$ Identityspecific goal representations in the lateral OFC can also be within a class of valued items. For example, in people who rated the value of 2 different foods as the same (e.g., milkshake and chocolate), the lateral OFC encoded these foods differently. ${ }^{106,107}$ Consistent with this finding, functional MRI showed that pleasant tastes of glucose compared to unpleasant salt tastes activated different areas of the OFC, suggesting that the OFC encodes both positive and negative affective valence..$^{51,108}$ The medial OFC encodes general reward value, such that food rated equally in value is encoded as equal representations. ${ }^{109}$ For example, in people who rated 2 different foods as the same (e.g., milkshake and chocolate), the medial OFC encoded these values as the same. ${ }^{109}$ This anatomic differentiation may help fine-tune food value and rewardencoding. When hungry, lean individuals chose a high-intensity, palatable odour compared with a different low-intensity, value-matched odour after devaluation via consumption of that food, they preferred the high-intensity odour of the nondevalued food. This odour-specific satiety was represented via the lateral posterior OFC, where pattern-based changes were found in functional MRI signals toward the sated odour, ${ }^{110}$ demonstrating the role of the OFC in encoding flexible representations of rewards via devaluation. ${ }^{51}$ Similar to obese rodents, obese humans also have deficits in satietyinduced outcome devaluation: a higher BMI is associated with reduced reward devaluation. ${ }^{89}$ Future research should assess whether this impaired reward devaluation in obese people is identity-specific or a general devaluation of rewards. Taken together, these findings suggest that obesity is associated with heightened response in the OFC to food and food cues, as well as reduced reward devaluation, and thus may be implicated in impairments in flexible food representations and behaviour.

\section{Executive function and obesity in humans}

Neurocognitive tests can be correlated with weight gain and obesity, and many studies have tried to uncover the genetic ${ }^{111,112}$ and behavioural links that can lead to overeating. ${ }^{113}$ Self-reported impulsivity traits have been positively correlated with caloric consumption. ${ }^{114}$ Executive control can be broadly defined as flexible, goal-directed behaviour and can be further broken down into response inhibition, attention shifting and working memory. Multiple groups have reviewed the correlation between obesity and executive function (see Valnik and colleagues ${ }^{20}$ and Yang and colleagues ${ }^{113}$ ). Here, we will focus on OFC-dependent response inhibition tasks.

The OFC plays an important role in response inhibition in multiple tasks and disorders. ${ }^{22,115}$ In the Stroop task, participants must name the word colour while ignoring what word is written (example: "red" written in green ink). The OFC is activated during response inhibition, ${ }^{116}$ and manipulations of the OFC via direct-current transcranial stimulation improve performance on the Stroop task. ${ }^{117}$ Performance on the Stroop task was also correlated with BMI: people with a higher BMI showed decreased response inhibition, ${ }^{90}$ and better scores were associated with weight loss. ${ }^{118}$ Furthermore, an increase in BMI is associated with decreased prefrontal and cingulate gyrus metabolic activity, which in turn is associated with poorer Stroop task scores. ${ }^{91}$ In a go/no-go task modified to include food cues, obese people made more errors than lean controls, ${ }^{92}$ and this finding was correlated with reduced activation of the OFC during this task in obese people compared with lean controls. ${ }^{104}$ The OFC may be a critical 
area for neuromodulation or other therapeutic intervention to improve impulse control and cognitive flexibility in obese people.

\section{OFC and binge-eating disorder}

Binge-eating disorder is the most prevalent specific eating disorder, and it is associated with obesity, although many individuals with binge-eating disorder are not obese. ${ }^{119}$ Recent studies have compared brain structure, neurochemistry and function in people with binge-eating disorder and obesity and in people without obesity (reviewed in Balodis and colleagues ${ }^{120}$ ). In a preliminary report, obese people with bingeeating disorder showed increased BOLD activity in the OFC in response to pictures of binge-type foods compared to nonfood stimuli (Table 1). ${ }^{93}$ This finding was consistent with those of other studies showing an increase in BOLD activity in the medial OFC in response to food cues and a positive association between the severity of binge-eating disorder and OFC activation. ${ }^{94,95}$ In these studies, the authors were unable to parse out differences in OFC activation between lean and obese people with binge-eating disorder, although changes in striatal function may distinguish obese people from those with binge-eating disorder. ${ }^{121}$ In other tasks, eating restraint in people with binge-eating disorder has been inversely associated with activity in the $\mathrm{OFC}$, anterior cingulate cortex and ventral medial PFC during a food Stroop task. ${ }^{97}$ Binge-eating disorder and bulimia nervosa are associated with increased OFC and anterior cingulate cortex grey matter volume compared with lean controls. ${ }^{96}$ It is possible that alteration in function of the OFC in people with binge-eating disorder may underlie dysfunctions in food reward processing and/ or controlled regulation of food intake. Thus, the OFC may be a viable target for neuromodulation in treatment of eating disorders.

\section{Conclusion}

The OFC integrates sensory and limbic cues to help guide feeding behaviour. ${ }^{22}$ A wealth of evidence indicates that neurocognitive measures for impulse control, decisionmaking and reward valuation are associated with eating behaviour and BMI. ${ }^{20}$ Interestingly, some of the genetic factors associated with obesity may underlie different neurocognitive endophenotypes in obese people. ${ }^{111}$ Many of these neurocognitive endophenotypes, such as impaired cognitive control of response inhibition regarding food intake, are associated with OFC function, ${ }^{86,113}$ supporting the notion that the OFC is critically implicated in obesity. In rodent studies, palatable or energy-dense diets impair outcome devaluation, stimulus-response associations and the selection of actions based on expected food value or the presence of food-paired cues. Future work should focus on the impact of palatable, obesogenic diets on mechanistic changes in OFC function to further understand how the OFC adapts to the obese state and how this may lead to further food intake. Furthermore, the OFC may be a valid target for therapeutic intervention in people with obesity for weight loss or uncontrolled eating.
Affiliations: From the Department of Physiology and Pharmacology, University of Calgary, Calgary, Alta., Canada (Seabrook, Borgland).

Competing interests: None declared.

Contributors: Both authors participated equally in the conception of the article and analysis and interpretation of the data. Both authors wrote and reviewed the article, approved the final version for publication and certify that no other individuals not listed as authors have made substantial contributions to the paper.

\section{References}

1. Hales CM, Carroll MD, Fryar CD, et al. Prevalence of obesity among adults and youth: United States, 2015-2016. NCHS Data Brief 2017;288:1-8

2. Ogden CL, Carroll MD, Fakhouri TH, et al. Prevalence of obesity among youths by household income and education level of head of household - United States 2011-2014. MMWR Morb Mortal Wkly Rep 2018;67:186-9.

3. Ogden CL, Carroll MD, Fryar CD, et al. Prevalence of obesity among adults and youth: United States, 2011-2014. NCHS Data Brief 2015;219:1-8.

4. Apovian CM. Obesity: definition, comorbidities, causes, and burden. Am J Manag Care 2016;22:s176-85.

5. Finkelstein EA, Ruhm CJ, Kosa KM. Economic causes and consequences of obesity. Annu Rev Public Health 2005;26:239-57.

6. Swinburn B, Sacks G, Ravussin E. Increased food energy supply is more than sufficient to explain the US epidemic of obesity. Am J Clin Nutr 2009;90:1453-6.

7. Hill JO, Wyatt HR, Reed GW, et al. Obesity and the environment: Where do we go from here? Science 2003;299:853-5.

8. Krebs JR. The gourmet ape: evolution and human food preferences. Am J Clin Nutr 2009;90:707S-11S.

9. Cornell CE, Rodin J, Weingarten H. Stimulus-induced eating when satiated. Physiol Behav 1989;45:695-704.

10. Hommel JD, Trinko R, Sears RM, et al. Leptin receptor signaling in midbrain dopamine neurons regulates feeding. Neuron 2006; 51:801-10.

11. Lutter M, Nestler EJ. Homeostatic and hedonic signals interact in the regulation of food intake. J Nutr 2009;139:629-32.

12. Ahima RS, Saper CB, Flier JS, et al. Leptin regulation of neuroendocrine systems. Front Neuroendocrinol 2000;21:263-307.

13. Cone RD. Anatomy and regulation of the central melanocortin system. Nat Neurosci 2005;8:571-8.

14. Berthoud H-R. Metabolic and hedonic drives in the neural control of appetite: Who's the boss? Curr Opin Neurobiol 2011;21:888-96.

15. Berthoud H-R, Morrison C. The brain, appetite, and obesity. Annu Rev Psychol 2008;59:55-92.

16. Kenny PJ. Reward mechanisms in obesity: new insights and future directions. Neuron 2011;69:664-79.

17. Timper K, Brüning JC. Hypothalamic circuits regulating appetite and energy homeostasis: pathways to obesity. Dis Model Mech 2017;10:679-89.

18. Naef L, Pitman KA, Borgland SL. Mesolimbic dopamine and its neuromodulators in obesity and binge eating. CNS Spectr 2015; 20:574-83

19. Liu S, Globa AK, Mills F, et al. Consumption of palatable food primes food approach behavior by rapidly increasing synaptic density in the VTA. Proc Natl Acad Sci U S A 2016;113:2520-5.

20. Vainik U, Dagher A, Dubé L, et al. Neurobehavioural correlates of body mass index and eating behaviours in adults: a systematic review. Neurosci Biobehav Rev 2013;37:279-99.

21. Lowe CJ, Reichelt AC, Hall PA. The prefrontal cortex and obesity: a health neuroscience perspective. Trends Cogn Sci 2019;23:349-61.

22. Kringelbach ML, Rolls ET. The functional neuroanatomy of the human orbitofrontal cortex: evidence from neuroimaging and neuropsychology. Prog Neurobiol 2004;72:341-72.

23. Ongür D, Price JL. The organization of networks within the orbital and medial prefrontal cortex of rats, monkeys and humans. Cereb Cortex 2000;10:206-19.

24. Ray JP, Price JL. The organization of the thalamocortical connections of the mediodorsal thalamic nucleus in the rat, related to the ventral forebrain-prefrontal cortex topography. J Comp Neurol 1992; 323:167-97. 
25. Uylings HB, van Eden CG. Qualitative and quantitative comparison of the prefrontal cortex in rat and in primates, including humans. Prog Brain Res 1990;85:31-62.

26. Van De Werd HJJM, Rajkowska G, Evers P, et al. Cytoarchitectonic and chemoarchitectonic characterization of the prefrontal cortical areas in the mouse. Brain Struct Funct 2010;214:339-53.

27. Murphy MJM, Deutch AY. Organization of afferents to the orbitofrontal cortex in the rat. J Comp Neurol 2018;526:1498-526.

28. Kawaguchi Y, Kondo S. Parvalbumin, somatostatin and cholecystokinin as chemical markers for specific GABAergic interneuron types in the rat frontal cortex. J Neurocytol 2002;31:277-87.

29. Pinto L, Dan Y. Cell-type-specific activity in prefrontal cortex during goal-directed behavior. Neuron 2015;87:437-50.

30. Miyamae T, Chen K, Lewis DA, et al. Distinct physiological maturation of parvalbumin-positive neuron subtypes in mouse prefrontal cortex. I Neurosci 2017:37:4883-902.

31. Quirk MC, Sosulski DL, Feierstein CE, et al. A defined network of fast-spiking interneurons in orbitofrontal cortex: responses to behavioral contingencies and ketamine administration. Front Syst Neurosci 2009;3:13.

32. $\mathrm{Hu} \mathrm{H}$, Gan J, Jonas P. Interneurons. Fast-spiking, parvalbumin+ GABAergic interneurons: from cellular design to microcircuit function. Science 2014;345:1255263.

33. Bissonette GB, Schoenbaum G, Roesch MR, et al. Interneurons are necessary for coordinated activity during reversal learning in orbitofrontal cortex. Biol Psychiatry 2015;77:454-64.

34. Thompson JL, Drysdale M, Baimel C, et al. Obesity-induced structural and neuronal plasticity in the lateral orbitofrontal cortex. Neuropsychopharmacology 2017;42:1480-90.

35. Reichelt AC, Killcross S, Hambly LD, et al. Impact of adolescent sucrose access on cognitive control, recognition memory, and parvalbumin immunoreactivity. Learn Mem 2015;22:215-24.

36. Reichelt AC, Gibson GD, Abbott KN, et al. A high-fat high-sugar diet in adolescent rats impairs social memory and alters chemical markers characteristic of atypical neuroplasticity and parvalbumin interneuron depletion in the medial prefrontal cortex. Food Funct 2019;10:1985-98.

37. Baker KD, Reichelt AC. Impaired fear extinction retention and increased anxiety-like behaviours induced by limited daily access to a high-fat/high-sugar diet in male rats: implications for dietinduced prefrontal cortex dysregulation. Neurobiol Learn Mem 2016;136:127-38

38. Sandoval-Salazar C, Ramírez-Emiliano J, Trejo-Bahena A, et al. A high-fat diet decreases GABA concentration in the frontal cortex and hippocampus of rats. Biol Res 2016;49:15.

39. Reichelt AC, Hare DJ, Bussey TJ, et al. Perineuronal nets: plasticity, protection, and therapeutic potential. Trends Neurosci 2019; 42:458-70.

40. Dingess PM, Harkness JH, Slaker M, et al. Consumption of a highfat diet alters perineuronal nets in the prefrontal cortex. Neural Plast 2018;2018:2108373.

41. Dingess PM, Darling RA, Kurt Dolence E, et al. Exposure to a diet high in fat attenuates dendritic spine density in the medial prefrontal cortex. Brain Struct Funct 2017;222:1077-85.

42. Schoenbaum G, Chiba AA, Gallagher M. Orbitofrontal cortex and basolateral amygdala encode expected outcomes during learning. Nat Neurosci 1998;1:155-9.

43. Schoenbaum G, Setlow B, Nugent SL, et al. Lesions of orbitofrontal cortex and basolateral amygdala complex disrupt acquisition of odorguided discriminations and reversals. Learn Mem 2003;10:129-40.

44. Takahashi YK, Roesch MR, Stalnaker TA, et al. The orbitofrontal cortex and ventral tegmental area are necessary for learning from unexpected outcomes. Neuron 2009;62:269-80.

45. Tremblay L, Schultz W. Relative reward preference in primate orbitofrontal cortex. Nature 1999;398:704-8.

46. Gutierrez R, Carmena JM, Nicolelis MAL, et al. Orbitofrontal ensemble activity monitors licking and distinguishes among natural rewards. J Neurophysiol 2006;95:119-33.

47. Namboodiri VMK, Otis JM, van Heeswijk K, et al. Single-cell activity tracking reveals that orbitofrontal neurons acquire and maintain a long-term memory to guide behavioral adaptation. Nat Neurosci 2019;22:1110

48. Jennings $\mathrm{JH}$, Kim $\mathrm{CK}$, Marshel JH, et al. Interacting neural ensembles in orbitofrontal cortex for social and feeding behaviour. Nature 2019;565:645-9.
49. Critchley HD, Rolls ET. Hunger and satiety modify the responses of olfactory and visual neurons in the primate orbitofrontal cortex. J Neurophysiol 1996;75:1673-86.

50. de Araujo IE, Gutierrez R, Oliveira-Maia AJ, et al. Neural ensemble coding of satiety states. Neuron 2006;51:483-94.

51. O'Doherty J, Rolls E, Francis S, et al. Sensory-specific satiety-related olfactory activation of the human orbitofrontal cortex. Neuroreport 2000;11:893-7.

52. Hikosaka K, Watanabe M. Delay activity of orbital and lateral prefrontal neurons of the monkey varying with different rewards. Cereb Cortex 2000;10:263-71.

53. Mori K, Manabe H, Narikiyo K, et al. Olfactory consciousness and gamma oscillation couplings across the olfactory bulb, olfactory cortex, and orbitofrontal cortex. Front Psychol 2013;4:743.

54. Fonseca E, de Lafuente V, Simon SA, et al. Sucrose intensity coding and decision-making in rat gustatory cortices. eLife 2018;7:pii: e41152.

55. Price JL. Definition of the orbital cortex in relation to specific connections with limbic and visceral structures and other cortical regions. Ann N Y Acad Sci 2007;1121:54-71.

56. Lichtenberg NT, Pennington ZT, Holley SM, et al. Basolateral amygdala to orbitofrontal cortex projections enable cue-triggered reward expectations. J Neurosci 2017;37:8374-84.

57. Padoa-Schioppa C, Conen KE. Orbitofrontal cortex: a neural circuit for economic decisions. Neuron 2017;96:736-54.

58. Pascoli V, Hiver A, Van Zessen R, et al. Stochastic synaptic plasticity underlying compulsion in a model of addiction. Nature 2018; 564:366-71.

59. Schoenbaum G, Roesch MR, Stalnaker TA, et al. A new perspective on the role of the orbitofrontal cortex in adaptive behaviour. Nat Rev Neurosci 2009;10:885-92.

60. Fettes P, Schulze L, Downar J. Cortico-striatal-thalamic loop circuits of the orbitofrontal cortex: promising therapeutic targets in psychiatric illness. Front Syst Neurosci 2017;11:25.

61. Gremel CM, Costa RM. Orbitofrontal and striatal circuits dynamically encode the shift between goal-directed and habitual actions. Nat Commun 2013;4:2264.

62. Gremel CM, Chancey JH, Atwood BK, et al. Endocannabinoid modulation of orbitostriatal circuits gates habit formation. Neuron 2016;90:1312-24.

63. Lu MT, Preston JB, Strick PL. Interconnections between the prefrontal cortex and the premotor areas in the frontal lobe. J Comp Neurol 1994;341:375-92.

64. Peak J, Hart G, Balleine BW. From learning to action: the integration of dorsal striatal input and output pathways in instrumental conditioning. Eur J Neurosci 2019;49:658-71.

65. McDannald MA, Jones JL, Takahashi YK, et al. Learning theory: a driving force in understanding orbitofrontal function. Neurobiol Learn Mem 2014;108:22-7.

66. Everitt BJ, Robbins TW. Neural systems of reinforcement for drug addiction: from actions to habits to compulsion. Nat Neurosci 2005; 8:1481-9.

67. Gillan CM, Papmeyer M, Morein-Zamir S, et al. Disruption in the balance between goal-directed behavior and habit learning in obsessive-compulsive disorder. Am J Psychiatry 2011;168: 718-26.

68. Volkow ND, Wise RA. How can drug addiction help us understand obesity? Nat Neurosci 2005;8:555-60.

69. Ramírez-Lugo L, Peñas-Rincón A, Ángeles-Durán S, et al. Choice behavior guided by learned, but not innate, taste aversion recruits the orbitofrontal cortex. J Neurosci 2016;36:10574-83.

70. Rudebeck PH, Saunders RC, Prescott AT, et al. Prefrontal mechanisms of behavioral flexibility, emotion regulation and value updating. Nat Neurosci 2013;16:1140-5.

71. Gallagher M, McMahan RW, Schoenbaum G. Orbitofrontal cortex and representation of incentive value in associative learning. J Neurosci 1999;19:6610-4.

72. Izquierdo A. Functional heterogeneity within rat orbitofrontal cortex in reward learning and decision making. J Neurosci 2017;37: 10529-40.

73. Wright SM, Aronne LJ. Causes of obesity. Abdom Imaging 2012; 37:730-2.

74. Kim J, Ragozzino ME. The involvement of the orbitofrontal cortex in learning under changing task contingencies. Neurobiol Learn Mem 2005;83:125-33. 
75. Kimchi EY, Torregrossa MM, Taylor JR, et al. Neuronal correlates of instrumental learning in the dorsal striatum. J Neurophysiol 2009; 102:475-89.

76. Yin HH, Knowlton BJ, Balleine BW. Lesions of dorsolateral striatum preserve outcome expectancy but disrupt habit formation in instrumental learning. Eur J Neurosci 2004;19:181-9.

77. Yin HH, Knowlton BJ, Balleine BW. Inactivation of dorsolateral striatum enhances sensitivity to changes in the action-outcome contingency in instrumental conditioning. Behav Brain Res 2006; 166:189-96.

78. Yin HH, Ostlund SB, Knowlton BJ, et al. The role of the dorsomedial striatum in instrumental conditioning. Eur J Neurosci 2005; 22:513-23.

79. Gallo M, Roldan G, Bures J. Differential involvement of gustatory insular cortex and amygdala in the acquisition and retrieval of conditioned taste aversion in rats. Behav Brain Res 1992;52:91-7.

80. Gardner MPH, Conroy JS, Shaham MH, et al. Lateral orbitofrontal inactivation dissociates devaluation-sensitive behavior and economic choice. Neuron 2017;96:1192-1203.

81. Reichelt AC, Morris MJ, Westbrook RF. Cafeteria diet impairs expression of sensory-specific satiety and stimulus-outcome learning. Front Psychol 2014;5:852.

82. Raji CA, Ho AJ, Parikshak N, et al. Brain structure and obesity. Hum Brain Mapp 2010;31:353-64.

83. Walther K, Birdsill AC, Glisky EL, et al. Structural brain differences and cognitive functioning related to body mass index in older females. Hum Brain Mapp 2010;31:1052-64.

84. Cazettes F, Cohen JI, Yau PL, et al. Obesity-mediated inflammation may damage the brain circuit that regulates food intake. Brain Res 2011;1373:101-9.

85. Alkan A, Sahin I, Keskin L, et al. Diffusion-weighted imaging features of brain in obesity. Magn Reson Imaging 2008;26:446-50.

86. Pursey KM, Stanwell P, Callister RJ, et al. Neural responses to visual food cues according to weight status: a systematic review of functional magnetic resonance imaging studies. Front Nutr 2014;1:7.

87. Stoeckel LE, Weller RE, Cook EW, et al. Widespread reward-system activation in obese women in response to pictures of high-calorie foods. Neuroimage 2008;41:636-47.

88. Burger KS, Stice E. Variability in reward responsivity and obesity: evidence from brain imaging studies. Curr Drug Abuse Rev 2011; 4:182-9.

89. Horstmann A, Dietrich A, Mathar D, et al. Slave to habit? Obesity is associated with decreased behavioural sensitivity to reward devaluation. Appetite 2015;87:175-83.

90. Gunstad J, Paul RH, Cohen RA, et al. Elevated body mass index is associated with executive dysfunction in otherwise healthy adults. Compr Psychiatry 2007;48:57-61

91. Volkow ND, Wang G-J, Telang F, et al. Inverse association between BMI and prefrontal metabolic activity in healthy adults. Obesity (Silver Spring) 2009;17:60-5.

92. Mobbs O, Iglesias K, Golay A, et al. Cognitive deficits in obese persons with and without binge eating disorder. Investigation using a mental flexibility task. Appetite 2011;57:263-71.

93. Geliebter A, Ladell T, Logan M, et al. Responsivity to food stimuli in obese and lean binge eaters using functional MRI. Appetite 2006;46:31-5

94. Filbey FM, Myers US, Dewitt S. Reward circuit function in high BMI individuals with compulsive overeating: similarities with addiction. Neuroimage 2012;63:1800-6.

95. Schienle A, Schäfer A, Hermann A, et al. Binge-eating disorder: reward sensitivity and brain activation to images of food. Biol Psychiatry 2009;65:654-61.

96. Schäfer A, Vaitl D, Schienle A. Regional grey matter volume abnormalities in bulimia nervosa and binge-eating disorder. Neuroimage 2010;50:639-43.

97. Balodis IM, Molina ND, Kober H, et al. Divergent neural substrates of inhibitory control in binge eating disorder relative to other manifestations of obesity. Obesity (Silver Spring) 2013;21:367-77.

98. Kosheleff AR, Araki J, Tsan L, et al. Junk food exposure disrupts selection of food-seeking actions in rats. Front Psychiatry 2018; 9:350.
99. Furlong TM, Jayaweera HK, Balleine BW, et al. Binge-like consumption of a palatable food accelerates habitual control of behavior and is dependent on activation of the dorsolateral striatum. J Neurosci 2014;34:5012-22.

100. Parkes SL, Furlong TM, Naneix F. Commentary: cafeteria diet impairs expression of sensory-specific satiety and stimulus-outcome learning. Front Psychol 2014;5:852.

101. Tantot F, Parkes SL, Marchand AR, et al. The effect of high-fat diet consumption on appetitive instrumental behavior in rats. Appetite 2017;108:203-11

102. Corbit LH, Balleine BW. Instrumental and Pavlovian incentive processes have dissociable effects on components of a heterogeneous instrumental chain. J Exp Psychol Anim Behav Process 2003; 29:99-106.

103. van der Laan LN, de Ridder DTD, Viergever MA, et al. The first taste is always with the eyes: a meta-analysis on the neural correlates of processing visual food cues. Neuroimage 2011;55:296-303.

104. Batterink L, Yokum S, Stice E. Body mass correlates inversely with inhibitory control in response to food among adolescent girls: an fMRI study. Neuroimage 2010;52:1696-703.

105. Rothemund Y, Preuschhof C, Bohner G, et al. Differential activation of the dorsal striatum by high-calorie visual food stimuli in obese individuals. Neuroimage 2007;37:410-21.

106. Suzuki S, Cross L, O'Doherty JP. Elucidating the underlying components of food valuation in the human orbitofrontal cortex. Nat Neurosci 2017;20:1780-6.

107. Klein-Flügge MC, Barron HC, Brodersen KH, et al. Segregated encoding of reward-identity and stimulus-reward associations in human orbitofrontal cortex. I Neurosci 2013;33:3202-11.

108. O'Doherty J, Rolls ET, Francis S, et al. Representation of pleasant and aversive taste in the human brain. J Neurophysiol 2001; 85:1315-21.

109. Howard JD, Gottfried JA, Tobler PN, et al. Identity-specific coding of future rewards in the human orbitofrontal cortex. Proc Natl Acad Sci U S A 2015;112:5195-200.

110. Howard JD, Kahnt T. Identity-specific reward representations in orbitofrontal cortex are modulated by selective devaluation. Neurosci 2017;37:2627-38

111. Vainik U, García-García I, Dagher A. Uncontrolled eating: a unifying heritable trait linked with obesity, overeating, personality and the brain. Eur J Neurosci 2019;50:2430-45.

112. Wood AC, Vainik U, Engelhardt LE, et al. Genetic overlap between executive functions and BMI in childhood. Am J Clin Nutr 2019; 110:814-22.

113. Yang Y, Shields GS, Guo C, et al. Executive function performance in obesity and overweight individuals: a meta-analysis and review. Neurosci Biobehav Rev 2018:84:225-44.

114. Guerrieri R, Nederkoorn C, Jansen A. How impulsiveness and variety influence food intake in a sample of healthy women. Appetite 2007;48:119-22.

115. Winstanley CA. The orbitofrontal cortex, impulsivity, and addiction. Ann N Y Acad Sci 2007;1121:639-55.

116. Konishi S, Nakajima K, Uchida I, et al. Common inhibitory mechanism in human inferior prefrontal cortex revealed by eventrelated functional MRI. Brain 1999;122:981-91.

117. Ouellet J, McGirr A, Van den Eynde F, et al. Enhancing decisionmaking and cognitive impulse control with transcranial direct current stimulation (tDCS) applied over the orbitofrontal cortex (OFC): a randomized and sham-controlled exploratory study. J Psychiatr Res 2015;69:27-34

118. Xu X, Deng Z-Y, Huang Q, et al. Prefrontal cortex-mediated executive function as assessed by Stroop task performance associates with weight loss among overweight and obese adolescents and young adults. Behav Brain Res 2017;321:240-8.

119. Kessler RC, Berglund PA, Chiu WT, et al. The prevalence and correlates of binge eating disorder in the World Health Organization World Mental Health Surveys. Biol Psychiatry 2013;73:904-14.

120. Balodis IM, Grilo CM, Potenza MN. Neurobiological features of binge eating disorder. CNS Spectr 2015;20:557-65.

121. Weygandt M, Schaefer A, Schienle A, et al. Diagnosing different binge-eating disorders based on reward-related brain activation patterns. Hum Brain Mapp 2012;33:2135-46. 